\title{
Distance and Rotational Speed Analysis of Coaxial Rotors for UTHM C-Drone
}

\author{
Che Muhammad Ikram Che Umar'1, Mohd Fadhli Zulkafli ${ }^{*}$
}

${ }^{1}$ Faculty of Mechanical and Manufacturing Engineering, Universiti Tun Hussein Onn Malaysia, Parit Raja, 86400, MALAYSIA

*Corresponding Author

DOI: https://doi.org/10.30880/paat.2021.01.01.006

Received 24 September 2021; Accepted 11 November 2021; Available online 21 December 2021

\begin{abstract}
The prototype of UTHM C-Drone use a coaxial hexacopter concept for its propulsion system. A coaxial rotor consists of two motor and two propellers mounted above each other and aligned in relation to their axis of rotation. The propellers are based on the T-Motor U15XXL KV29 model used in UTHM C-Drone. The distance between the two propellers is usually relative to the radius of the propeller or can be lesser. The objectives for this study are to investigate the effect of distance between upper and lower propeller in a coaxial rotors system and the effect of rotational speed. This study is important to ensure the C-Drone power efficient and capable to lift $180 \mathrm{~kg}$ payload. The CAD model of the propeller and coaxial rotors system were designed based on the specification from T-Motor company by Solidworks software and the flow simulations were conducted using Solidworks Flow Simulation module. The total of six CAD models; one for a single propeller and five for coaxial rotors with five difference of distance cases were constructed. For each model, the total thrust was tested from $50 \%$ throttle power up to the $90 \%$ throttle power. It was found that the coaxial rotors system can generate more thrust than a single propeller but less than double. It was also found that if the lower propeller rotates faster than the upper propeller, the increment of total thrust is very small. However, if the upper propeller rotates faster than the lower propeller, the total thrust increase significantly. For the case of faster upper propeller, as the higher the throttle applied, the thrust increment ratio will decrease, and the efficiency of the thrust produced will be affected. In addition, for same rotation speed, the thrust generated was lesser when both propellers rotate in a same direction compared to when each propeller rotates in the opposite directions of each other.
\end{abstract}

Keywords: Coaxial propeller, UAV, hexacopter, cargo drone

\section{Introduction}

Unmanned aerial vehicle (UAV), or known as a drone, is an aircraft where there is no onboard human who controls the aircraft and is either being controlled from the ground or automatically driven by some predetermined flight missions. Drones have been around for centuries and were solely used for military purposes [1] and have previously been used in military applications such as aerial recognition, monitoring and arms organization. Mostly, drones are always used for boring, dirty and dangerous work as stated in the primary function of the drone once suggested for the first time.

Over the years, the purposes of drones nowadays have developed significantly not just for military purposes but also for civil applications. Drones can be classified based on their usage such as for photography, aerial mapping, aerial platform, surveillance etc. However, the best drone classification can be made based on the aerial platform where it is classified into four major types of drones which are Multirotor Drone, Fixed Wing Drone, Single Rotor Helicopter and Fixed Wing Hybrid Vertical Take-off Landing (VTOL) [2]. Drones also can be classified into many categories according to the standard produce issued by the manufacturer. 
There are many active studies and developments on drones to be used as a medium to deliver cargo or parcels. A working prototype cargo drone unveiled by Boeing marks the entry of the world's largest aircraft manufacturer into the unmanned electric air cargo air vehicle space [3]. At the same time, Universiti Tun Hussein Onn Malaysia (UTHM) was also developing a multirotor cargo drone, named as C-Drone, that is expected to carry a payload up to $180 \mathrm{~kg}$ [4]. The UTHM C-Drone is classified as a hexacopter [5] with coaxial rotors as illustrated in Fig. 1. In other words, it is a multirotor drone that is lifted and propelled by twelve rotors. The benefit of using this configuration is it can generate more thrust and power where it can contribute to the capability of the drone to lift a heavy load. The 6 arms system or 12 rotors not only provides a more stable flight and more power but is also an important safety feature [6].

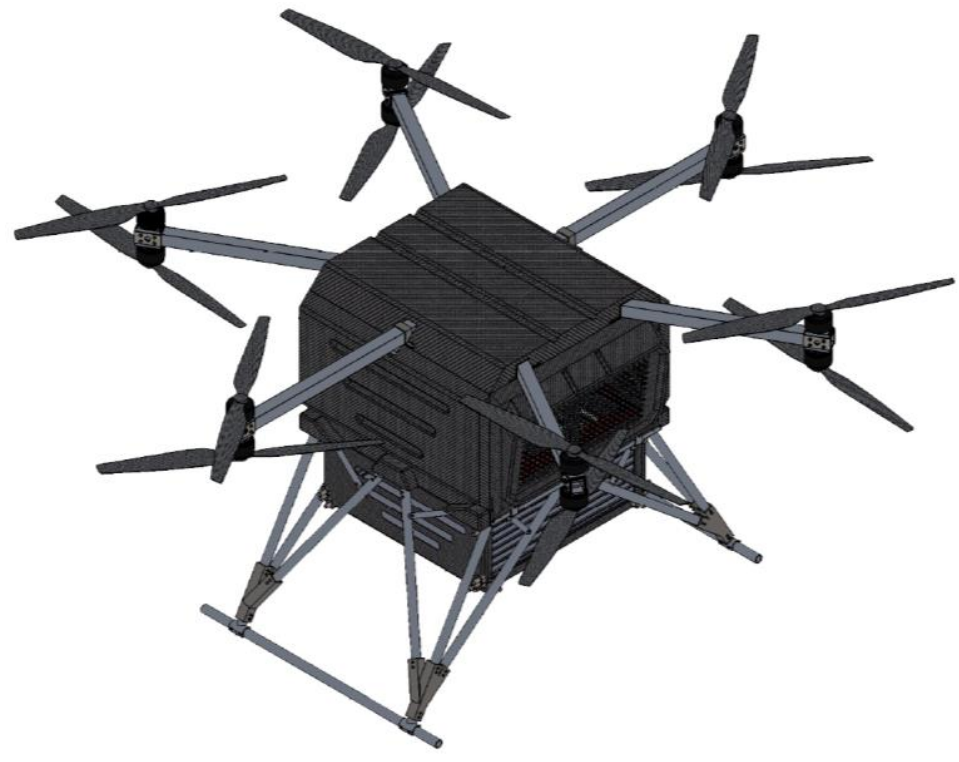

Fig. 1 - UTHM C-Drone

A coaxial rotors system composed of two engines and two propellers disposed one above the other and aligned in relation to their axis of rotation. In addition, there are many types of a helicopter using coaxial rotors. Helicopter with coaxial propulsion is special because it uses two contra-rotating rotors to compensate for each other's torque that they apply to the helicopter fuselage when they rotated [7]. One most often-cited advantage of the contra-rotating coaxial rotor design applied to a helicopter is that the overall rotor diameter can be reduced [8] and no tail rotor is required for antitorque and directional control [9]. Therefore, the same benefit in terms of rotor diameter reduction while being able to transport heavier payload can be obtained if the coaxial rotors system is applied to the multirotor drone.

Normally the distance between the two propellers is approximately equal to the radius of the propellers [10]. The distance between two propellers will affect the efficiency of the coaxial rotors system. The experiment reported by [11] does recommend that the ratio of distance to propeller radius should be more than 0.357 . With a coaxial hexacopter configuration, the overall rotor diameter can be reduced since the total thrust generated from all rotors contribute sufficient vertical thrust to overcome the weight of the drone. This study will be focusing on the distance between the two propellers and the propeller rotation speed of the coaxial rotors since the efficiency of power required of the C-Drone is related to the optimum configuration of its propulsion system.

\section{Methodology}

\subsection{Computational Model}

Since the C-Drone uses the 62-inch (or approximately $1.58 \mathrm{~m}$ ) diameter propellers for U15XXL KV29 T-Motor [12], the propeller is redesigned in the Computational Aided Design (CAD) software, i.e. Solidwork, as shown in Fig. 2, based on the specification provided by the T-Motor manufacturer [12]. At full power, the manufacturer reported that the 64-inch propeller will rotate at $2245 \mathrm{RPM}$ and capables to generate $102.354 \mathrm{~kg}$ of thrust. In other to compare the thrust of the coaxial rotors system with a single propeller, the case of a single propeller also was being simulated in the flow simulation.

The CAD design is required for the flow simulation to determine the capability of the coaxial rotors to produce thrust according to the distance between two propellers. In this study, the distance between the upper and lower propeller is defined as $h$ and the propeller radius is $r_{p}$ in Fig. 3. Five different cases of $h$ were tested in this study; $200 \mathrm{~mm}, 300 \mathrm{~mm}$, $400 \mathrm{~mm}, 500 \mathrm{~mm}$ and the distance recommended by [11] which is $0.357 r_{p}$ or equivalent to $282 \mathrm{~mm}$ for the 62 -inch diameter propeller. Therefore, there are a total of six CAD models were constructed in this study: a single propeller model and the five coaxial rotors model with different distances between its propellers. 


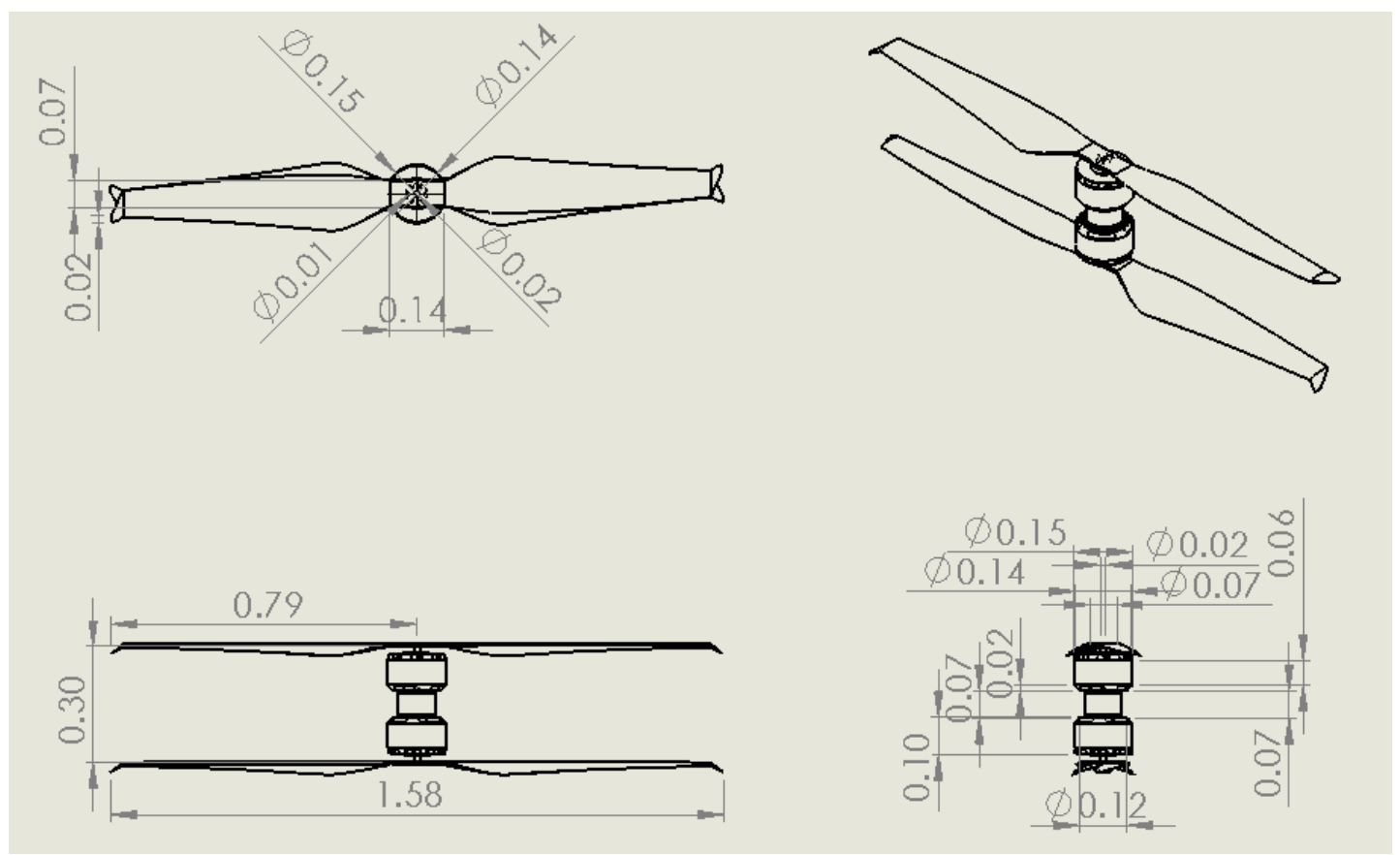

Fig. 2 - Configuration and dimension of the C-Drone coaxial rotors

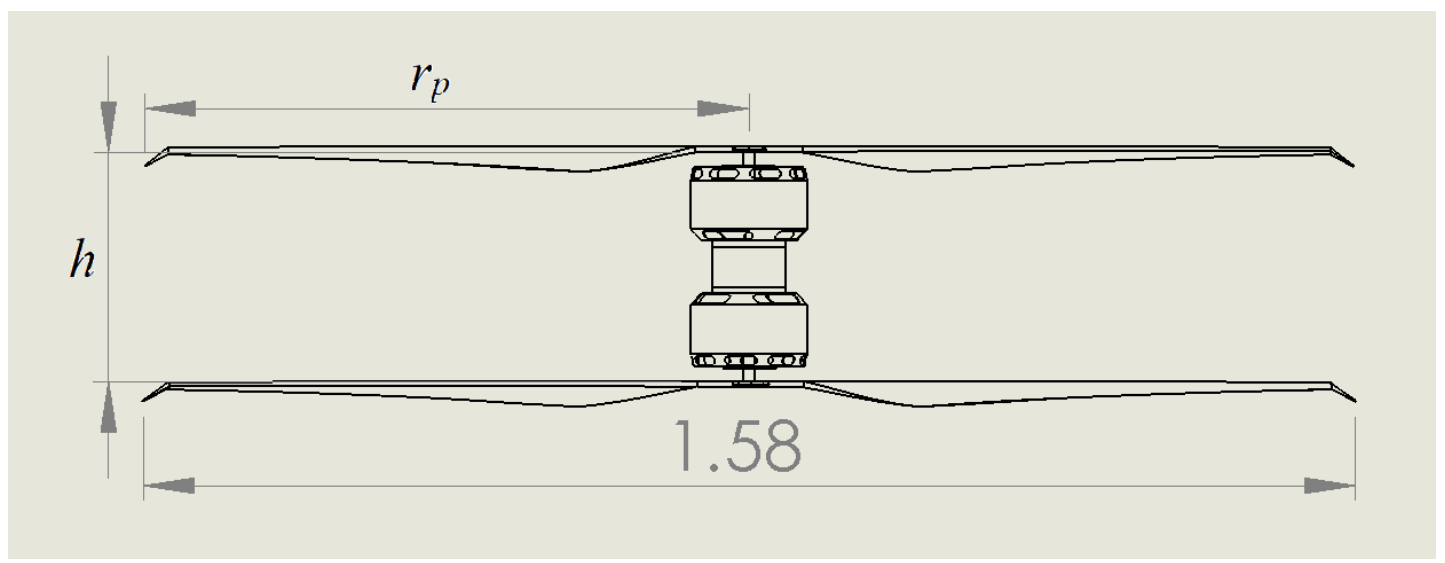

Fig. 3 - Geometry parameters of a coaxial propeller

Solidworks Flow Simulation module that is embedded within the Solidwork software was used to conduct the flow simulation through Computational Fluid Dynamics (CFD) method. The module enables a user to simply simulate air flows through and around the rotating coaxial rotors model to approximate the thrust that can be generated from the model. Before generating a mesh for the flow simulation, the CFD computational domain must be defined around the CAD model.

There are two kinds of computational domains created in this study. The first domain is a static region while the second domain is defined as a rotating region. In the rotating region, the Solidwork Flow Simulation module uses the Moving Reference Frame (MRF) technique to simulate the effect of rotating machinery, which in this study is the propellers. This technique is a simple and efficient steady-state CFD method that assumes the solution is in a steady state because the propellers' rotation and the assigned volume around the propellers can be considered constant over a longer time frame. MRF technique is extremely useful to save computational cost and time compared to other alternatives such as the dynamic or sliding mesh method.

In the Solidwork Flow Simulation module, a transparent box or an enclosure was mounted automatically upon completion of all the necessary parameters set up for the flow simulation. This enclosure is the static computational domain. As shown in Fig. 4, the static computational domain is the rectangular parallel-piped section where the 3D flow analysis calculations were executed. As the external flow type of analysis is selected in the Solidwork Flow Simulation module, boundary planes of the static computational domain are automatically distanced from the rotational computational domain. 
Meanwhile, the rotating region was created for each propeller since the propeller can be rotating in the opposite direction of each other. By creating the local rotating region, the fragment of the rotating region is only propellers out of whole components in the assembly. The rotating region generally assumes that a model is completely symmetrical about the rotating axis and that all components within the computational domain rotate at the speed specified on the rotating reference frame. In this study, the positive value of angular velocity indicates the counter-clockwise direction meanwhile a negative value of angular velocity specifies the clockwise direction of rotation.

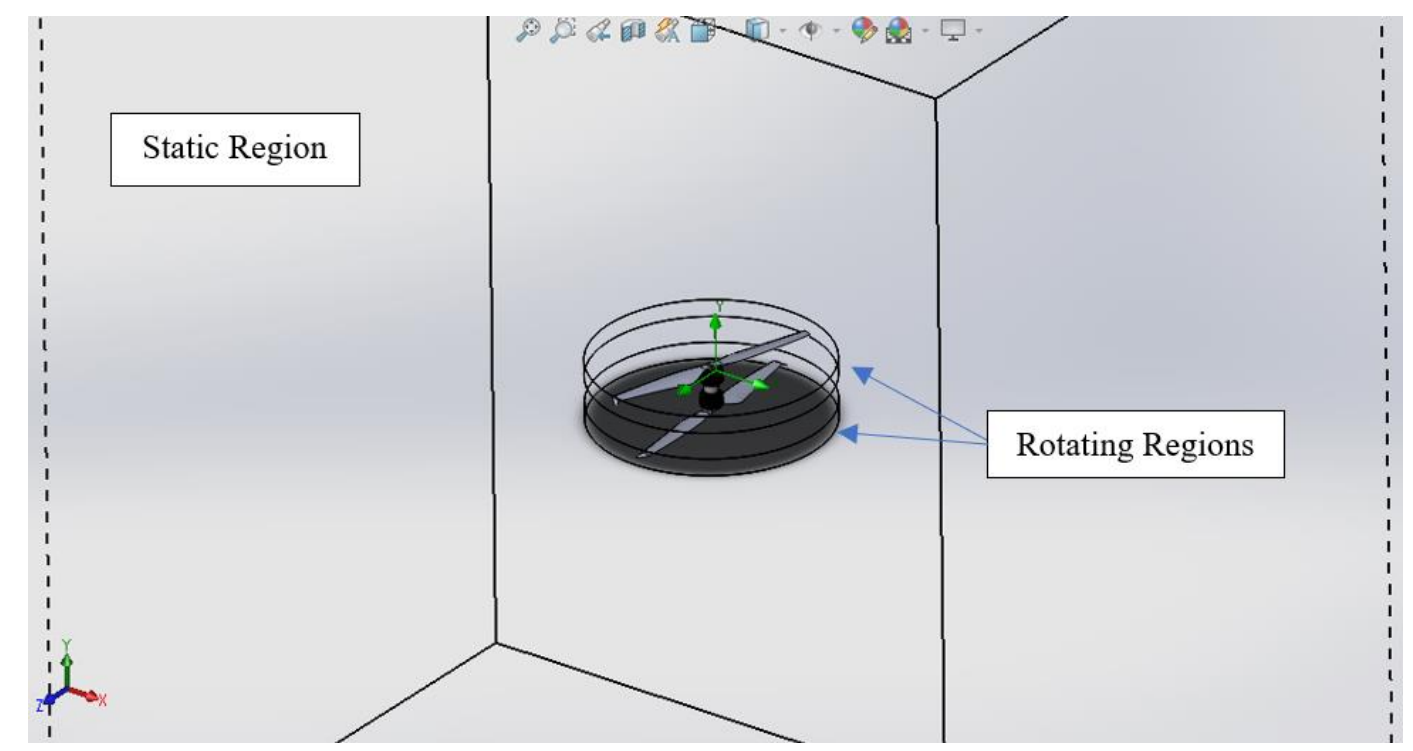

Fig. 4 - Computational domain in Solidworks Flow Simulation module

\subsection{Computational Mesh}

Meshing is a distinct representation of the geometry involved in the problem and is to be solved computationally. Fundamentally, in order to analyses fluid flows, flow domains are split into smaller subdomains. The governing equations, i.e. Navier-stokes equation, are then discretized and solved inside each of these subdomains. In addition, Solidworks Flow Simulation used the Favre-averaged Navier-Stokes equations with $k-\varepsilon$ model to predict turbulent flows.

Solidworks Flow Simulation use the Immersed Body Structured Cartesian Mesh, or simply known as the Cartesian Mesh approach. In this approach, the mesh generation begins with dividing the computational domain into a set of cuboid cells and then be refined near the CAD models to resolve features such as curvature surfaces and narrow channels.
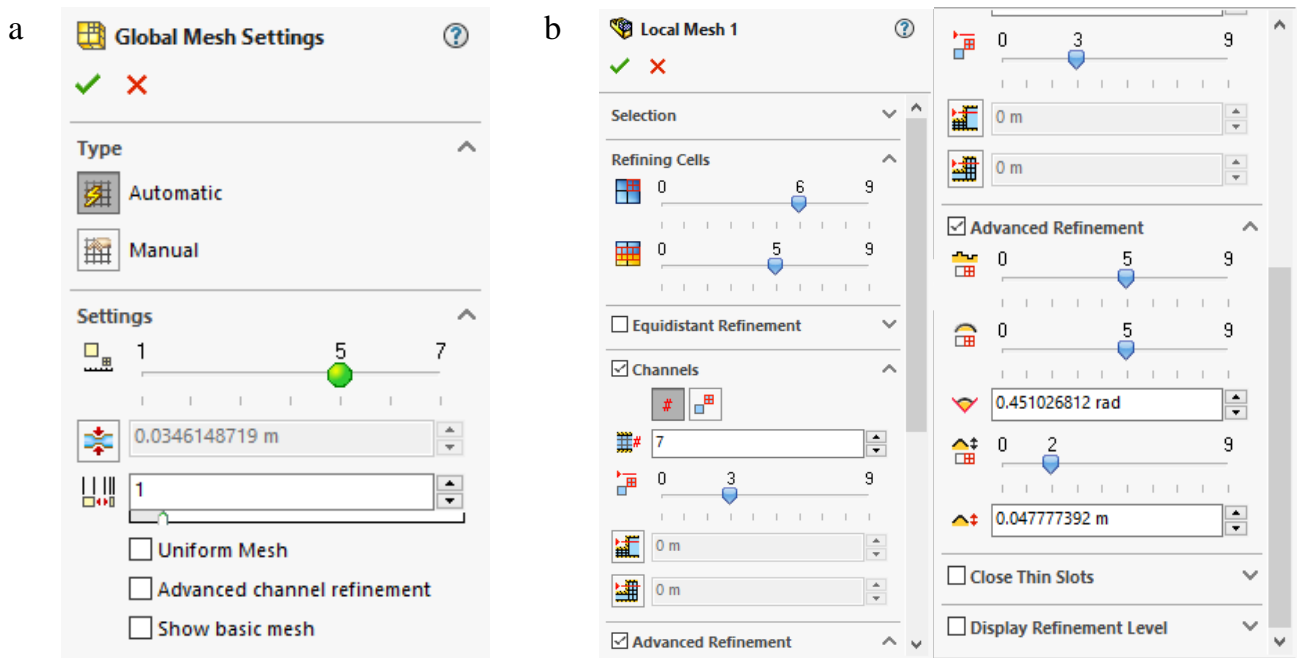

Fig. 5 - (a) The global mesh settings dialogue; (b) The local mesh settings dialogue

Solidworks Flow Simulation module offers an automated meshing process for the whole computational domain, i.e. the global mesh, by default unless a manual type of meshing is picked. The global mesh setting for this study is as shown in Fig. 5(a). Under automatic meshing, the level of initial mesh which governs the number of basic mesh cells can be varied from scale 1 to 7 . A higher scale of meshing means a smaller size of cuboid cells which results in a higher number 
of mesh globally. In this study, the level of initial mesh is set as scale 5. The resulting global mesh is illustrated in Fig. 6.

Meanwhile, the local mesh setting shown in Fig. 5(b) was used in addition to global meshing for mesh refinement around the propeller and the coaxial rotors CAD model. The resulting meshes are illustrated in Fig. 7. The local mesh allows defining an initial mesh in a local area of the computational domain to enhance the model-specific geometry and flow characteristics within this field, which cannot be well solved under global initial mesh settings. The selection of components for the local mesh was the region of the propeller and also the rotating region. These control settings of meshes are important because it determines the mesh quality which results in the accuracy of the calculated flow simulation.

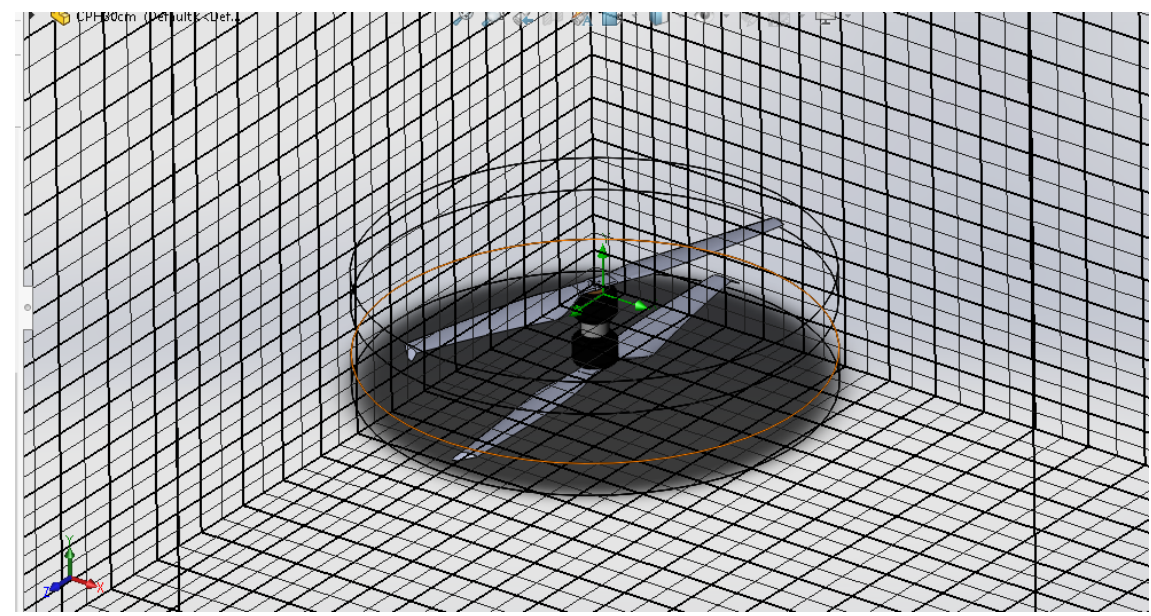

Fig. 6 - Cartesian mesh generated from the global mesh setting

a

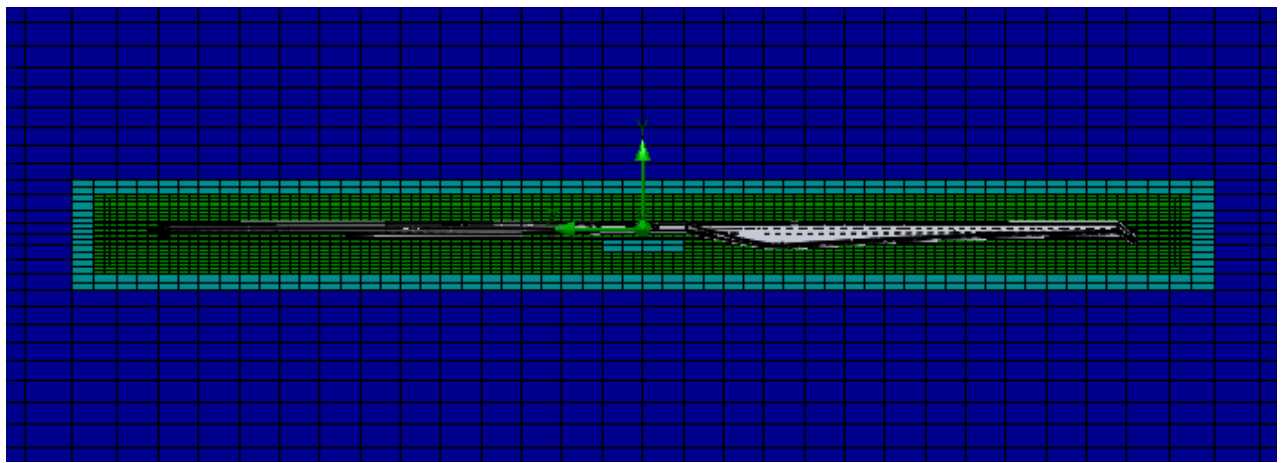

$\mathrm{b}$

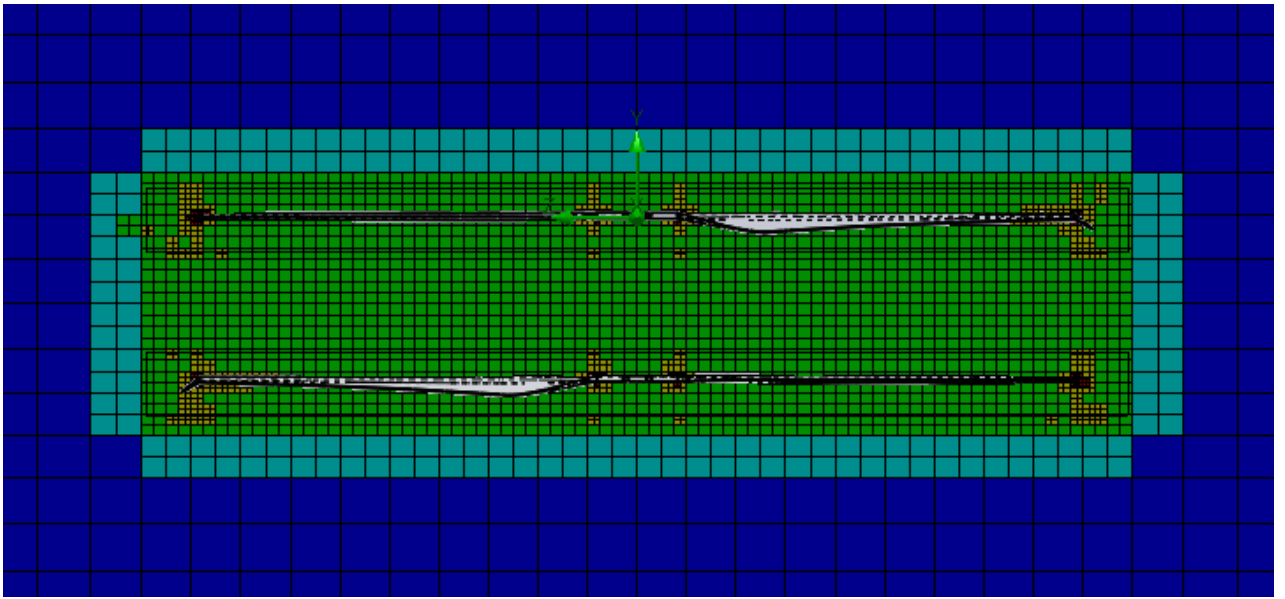

Fig. 7 - Cartesian mesh generated on (a) Single Propeller; (b) Coaxial Rotors 


\section{Results and Discussion}

\subsection{Validation with Manufacturer Specification}

The thrust production test for the designed single propeller was carried out in the flow simulation. The initial percentage of the throttle used to measure the thrust in this flow simulation was $50 \%$ of full power because the $50 \%$ throttle was the ideal power to hover a drone. Then, the throttle was increased by the interval of $10 \%$ up to $90 \%$ throttle. Meanwhile, the actual thrust for U15XXL KV29 T-Motor propeller is given from the specification sheet [12] that has been provided by the T-Motor Company.

The comparison of simulations' results with the actual thrust obtained from the manufacturer is shown in Table 1. Quantitatively, there are around $40 \%$ differences on average between the thrust obtained from the simulation and the actual thrust provided by the manufacturer. Nevertheless, the trend is the same. As the throttle increases, the rate of thrust increment become smaller. As the focus of this study is to find the optimum distance that can provide the most thrust, the accurate value of thrust is not the main objective but the comparison of thrust generated from different cases is more important. It is expected that the thrust value will not be accurate, but the trend will be the same as the actual model.

Table 1 - Comparison of U15XXL KV29 Propeller's simulation and actual thrust

\begin{tabular}{|c|c|c|c|c|c|}
\hline \multirow{2}{*}{ Throttle (\%) } & \multirow{2}{*}{ RPM } & \multicolumn{2}{|c|}{ Simulation } & \multicolumn{2}{|c|}{ Actual } \\
\hline & & Thrust (N) & Increment & Thrust (N) & Increment \\
\hline 50 & 1371 & 210.24 & - & 358.21 & - \\
\hline 60 & 1580 & 295.99 & $41 \%$ & 482.90 & $35 \%$ \\
\hline 70 & 1771 & 370.12 & $25 \%$ & 613.80 & $27 \%$ \\
\hline 80 & 1946 & 446.01 & $21 \%$ & 746.72 & $22 \%$ \\
\hline 90 & 2104 & 519.65 & $17 \%$ & 877.84 & $18 \%$ \\
\hline
\end{tabular}

\subsection{Thrust based on Upper and Lower Rotor Distance}

The thrust values obtained from the flow simulation are recorded according to the propellers' clearance distance and throttle percentage or rotational speed. In this section, both upper and propellers are rotating at the same speed but in the opposite direction of each other. The results are shown in 3 separate tables which show the thrust generated by the upper propeller (Table 2), lower propeller (Table 3) and the total thrust generated by the coaxial rotors configuration (Table 4).

As the distance between upper and lower propellers of coaxial rotors system increases, overall the thrust of the upper propeller increases while the lower propeller decreases. However, due to the higher rate of thrust increment of the upper propeller, as listed in Table 4, the total thrust generated by the coaxial rotors increase as the distance increases. Based on Table 4, Fig. 8 plotted the comparison of thrusts generated from the different distances of the coaxial rotors and single propeller against its rotational speed in RPM, which is related to the throttle percentage. The coaxial rotors system does provide higher thrust compared to a single propeller, but the amount is less than double of a single propeller thrust. Moreover, the effect of distance become noticeable at a rotational speed larger than 1800 RPM as the thrust of the upper propeller rise more rapidly.

Table 2 - Thrust for upper propeller

\begin{tabular}{cccccc}
\hline \multirow{2}{*}{ Throttle (\%) } & \multicolumn{5}{c}{ Thrust (N) } \\
\cline { 2 - 6 } & $200 \mathrm{~mm}$ & $282 \mathrm{~mm}$ & $300 \mathrm{~mm}$ & $400 \mathrm{~mm}$ & $500 \mathrm{~mm}$ \\
\hline $\mathbf{5 0}$ & 211.6475 & 229.5427 & 230.7121 & 232.2068 & 249.9843 \\
$\mathbf{6 0}$ & 283.5497 & 305.9663 & 307.2226 & 285.7753 & 333.4917 \\
$\mathbf{7 0}$ & 343.8136 & 383.9251 & 386.6537 & 356.0311 & 420.643 \\
$\mathbf{8 0}$ & 411.1251 & 463.0485 & 464.7439 & 428.1524 & 498.6565 \\
$\mathbf{9 0}$ & 486.4666 & 541.4962 & 542.7725 & 547.087 & 584.1271 \\
\hline
\end{tabular}


Table 3 - Thrust for lower propeller

\begin{tabular}{cccccc}
\hline \multirow{2}{*}{ Throttle (\%) } & \multicolumn{5}{c}{ Thrust (N) } \\
\cline { 2 - 6 } & $200 \mathrm{~mm}$ & $282 \mathrm{~mm}$ & $300 \mathrm{~mm}$ & $400 \mathrm{~mm}$ & $500 \mathrm{~mm}$ \\
\hline $\mathbf{5 0}$ & 147.7429 & 133.0098 & 128.6742 & 156.7183 & 116.7479 \\
$\mathbf{6 0}$ & 203.0352 & 178.6679 & 175.2734 & 189.1883 & 152.6563 \\
$\mathbf{7 0}$ & 241.8260 & 224.3822 & 217.6034 & 235.7292 & 191.5811 \\
$\mathbf{8 0}$ & 280.9090 & 264.9797 & 255.1281 & 284.7612 & 283.8484 \\
$\mathbf{9 0}$ & 342.3841 & 314.2024 & 298.4941 & 367.5093 & 331.6835 \\
\hline
\end{tabular}

Table 4 - Thrust for coaxial rotor configuration

\begin{tabular}{cccccc}
\hline \multirow{2}{*}{ Throttle (\%) } & \multicolumn{5}{c}{ Thrust $\mathbf{( N )}$} \\
\cline { 2 - 6 } & $200 \mathrm{~mm}$ & $282 \mathrm{~mm}$ & $300 \mathrm{~mm}$ & $400 \mathrm{~mm}$ & $500 \mathrm{~mm}$ \\
\hline $\mathbf{5 0}$ & 359.3905 & 362.5525 & 359.3863 & 388.925 & 366.7322 \\
$\mathbf{6 0}$ & 486.5848 & 484.6342 & 482.496 & 474.9636 & 486.148 \\
$\mathbf{7 0}$ & 585.6397 & 608.3073 & 604.2571 & 591.7603 & 612.2241 \\
$\mathbf{8 0}$ & 692.0341 & 728.0281 & 719.8719 & 712.9135 & 782.5049 \\
$\mathbf{9 0}$ & 828.8507 & 855.6986 & 841.2666 & 914.5963 & 915.8106 \\
\hline
\end{tabular}

\section{Total Thrust Coaxial Configuration}

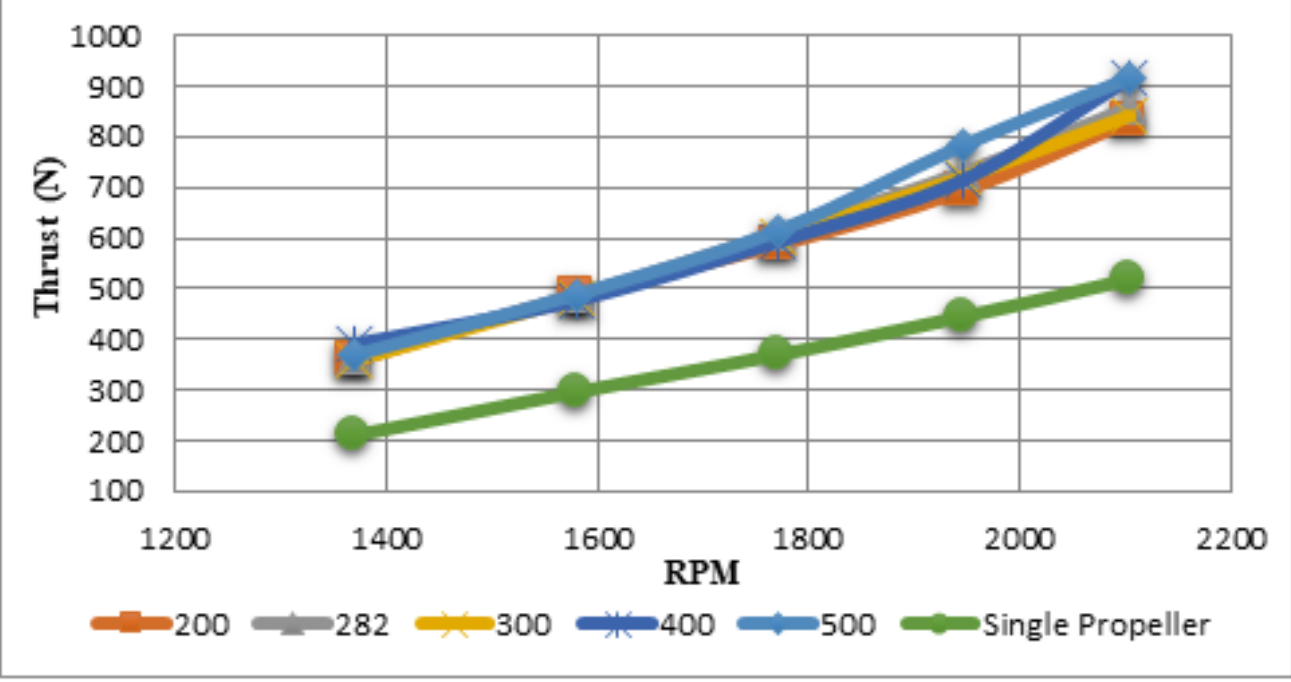

Fig. 8 - Comparison of coaxial rotors and single propeller thrust cases

By further investigation on the flow structure around the propellers, as shown in Fig. 9, the slipstream (the passing through flow) of the coaxial rotors is faster for $h=400 \mathrm{~mm}$ case compared to $h=200 \mathrm{~mm}$. If the resulting slipstream is fast compared to the incoming airflow, the thrust generated will become higher. As the distance becomes larger, the slipstream of the upper propeller will be less disturbed by the existence of a lower propeller hence the upper propeller can provide more thrust. However, this also generates more wake turbulence from the upper propeller. Therefore, the incoming flow to the lower propeller will be disturbed by this wake turbulence and become more severe as the distance between the upper and lower propeller increases. As a result, a lower propeller will generate less thrust. 
a

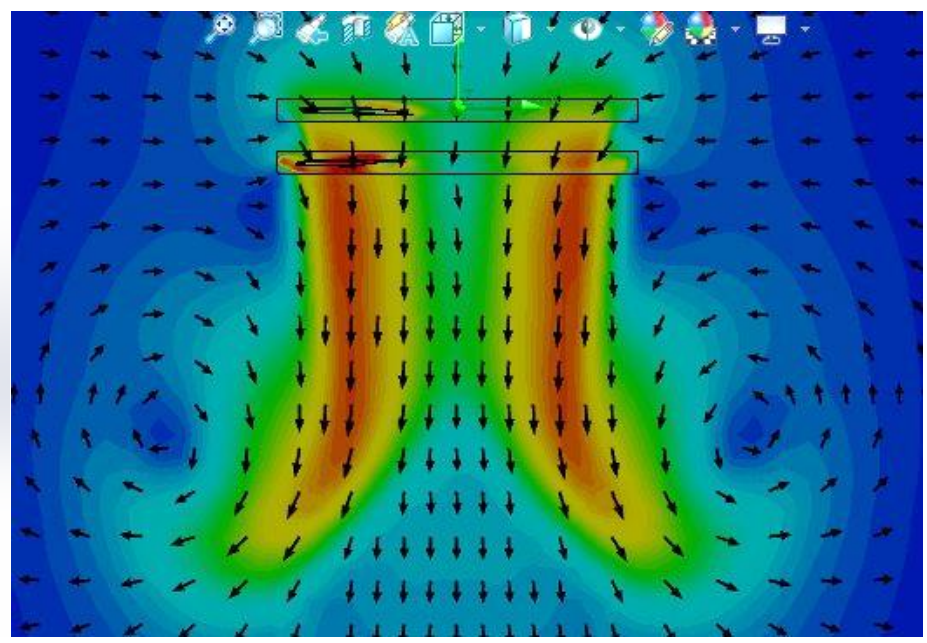

b

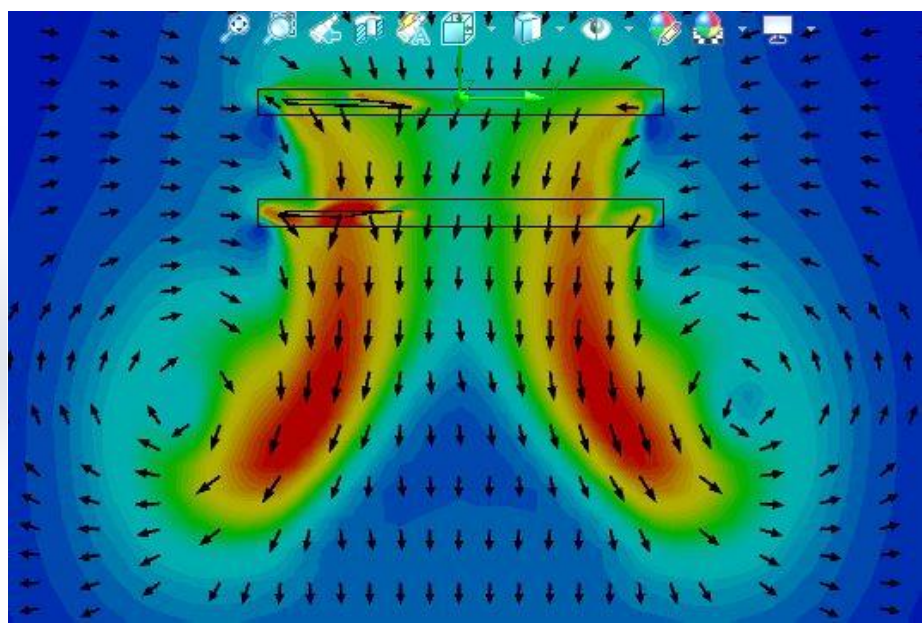

Fig. 9 - Velocity contour of the flow for (a) $200 \mathrm{~mm}$ distance; (b) $400 \mathrm{~mm}$ distance case (90\% throttle)

\subsection{Thrust of Coaxial Propeller with Different RPM}

Another simulation conducted is to calculate the thrust generated by the propellers with different rotational speeds between the upper propeller and lower propeller on the coaxial rotors. The results are shown in Table 5 and plotted in Fig. 7 for easy comparison. In Table 5, the negative value of rotational speed (RPM) indicates the opposite direction of the propeller compared to the positive value of RPM.

The differences of total thrust between the same rotational speed coaxial rotor and different rotational speeds of coaxial rotor are insignificant. However, it is found that if the lower propeller rotates faster than the upper propeller, the increment of total thrust is very small. In contrast, if the upper propeller rotates faster than the lower propeller, the total thrust increase significantly. Therefore, a major contribution of the thrust in the coaxial rotors system comes from the upper propeller. As there is a small difference in thrust for different speed coaxial rotors and same rotational speed coaxial rotors, the same rotational speed is recommended since the resultant moments from such configuration will be zero and hence ease the drone control system.

Table 5 - 282 mm clearance distance of Coaxial Propeller with different RPM

\begin{tabular}{ccccc}
\hline \multicolumn{2}{c}{ Rotational Speed (RPM) } & \multicolumn{3}{c}{ Thrust (N) } \\
\hline Upper Propeller & Lower Propeller & Upper Propeller & Lower Propeller & Total \\
\hline 1946 & -1946 & 404.2965 & 244.5660 & 648.8625 \\
1946 & -2027 & 410.5053 & 245.7892 & 656.2944 \\
2027 & -1946 & 506.1514 & 251.0476 & 757.1990 \\
2027 & -2027 & 503.4226 & 287.7127 & 791.1353 \\
\hline
\end{tabular}




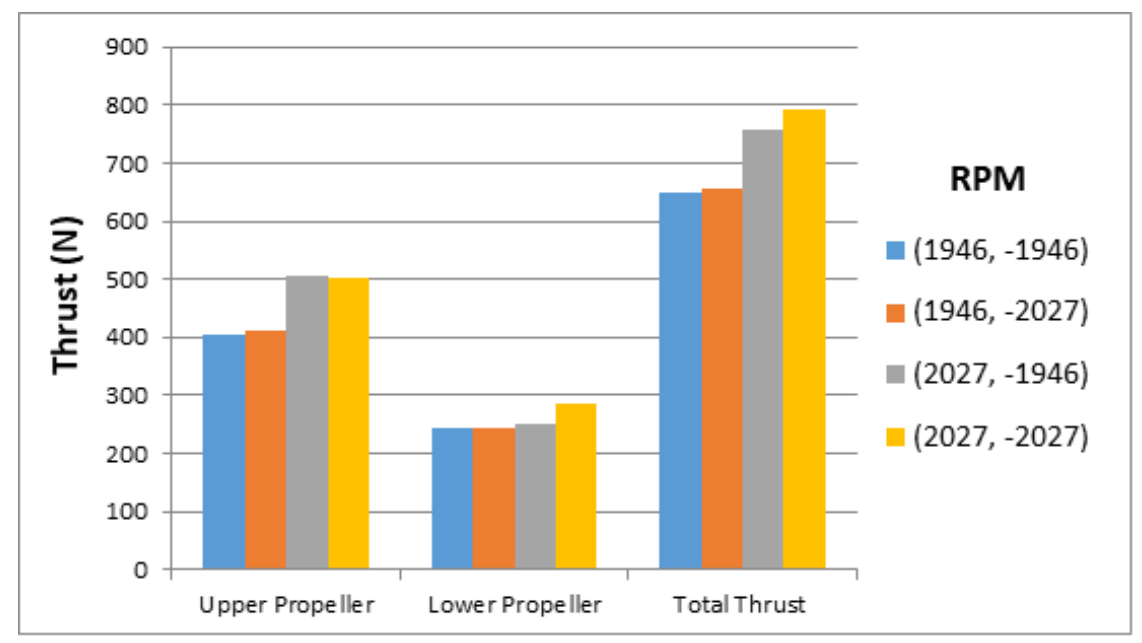

Fig. 10 - Graph of 282 mm Coaxial Propeller with Different RPM

In addition, the simulation of thrust increment ratio for different speed coaxial rotors is conducted. The thrust generated from the $282 \mathrm{~mm}$ clearance distance of the coaxial propeller was used to calculate the thrust increment ratio. The results of the thrust increment ratio are shown in Table 6 . The thrust increment ratio for $282 \mathrm{~mm}$ clearance distance of coaxial propeller decreases when the percentage of propeller's throttle increase. This show that the higher the throttle applied, the thrust increment ratio will decrease, and the efficiency of the thrust produced will be affected.

Table 6 - Thrust Increment Ratio of 282 mm Clearance Distance

\begin{tabular}{cc}
\hline Throttle Ratio & Thrust Increment Ratio \\
\hline $\mathbf{6 0 : 5 0}$ & 1.347 \\
$\mathbf{7 0 : 6 0}$ & 1.255 \\
$\mathbf{8 0 : 7 0}$ & 1.197 \\
$\mathbf{9 0 : 8 0}$ & 1.175 \\
\hline
\end{tabular}

Another calculation is by setting up the same rotational direction and speed of the upper propeller and lower propeller of the coaxial rotors. Only one distance is considered, which is $200 \mathrm{~mm}$, and the results are shown in Table 7. Compared with Table 4 for different direction propellers configuration, it was found that the lower propeller produces less thrust while the upper propeller gains slightly higher thrust for this configuration. Thus, the total thrust of the same rotational direction is overall lower than the opposite rotational direction of coaxial rotors.

Table 7 - $200 \mathrm{~mm}$ distance of coaxial rotors with the same rotational direction

\begin{tabular}{cccc}
\hline \multirow{2}{*}{ Throttle (\%) } & \multicolumn{3}{c}{ Thrust (N) } \\
\cline { 2 - 4 } & Upper Propeller & Lower Propeller & Total \\
\hline $\mathbf{5 0}$ & 209.6260 & 102.6666 & 312.2926 \\
$\mathbf{6 0}$ & 289.1621 & 151.7403 & 440.9023 \\
$\mathbf{7 0}$ & 360.4216 & 202.7662 & 563.1878 \\
$\mathbf{8 0}$ & 432.3217 & 243.6758 & 676.0029 \\
$\mathbf{9 0}$ & 496.5848 & 263.1818 & 759.7666 \\
\hline
\end{tabular}

\section{Conclusions}

This study is conducted for the development of UTHM C-Drone. The propellers and coaxial rotors system were designed using Solidwork software based on the 62-inch diameter propellers for U15XXL KV29 T-Motor because this is the system used for the UTHM C-Drone. As the distance between the upper and lower propeller of the coaxial rotors increases, the total thrust generated will increase but less than double of thrust generated by a single propeller. This is due to the suction region of the lower propeller being disturbed by the wake turbulence of the upper propeller, hence both propellers will not act as two independent systems. 
It was also found that if the lower propeller rotates faster than the upper propeller, the increment of total thrust is very small. However, if the upper propeller rotates faster than the lower propeller, the total thrust increase significantly. Moreover, for the case of the faster upper propeller, the higher the throttle applied, the thrust increment ratio will decrease, and the efficiency of the thrust produced will be affected. The same rotational speed of the upper and lower propeller is recommended since this will ease the control setting and prevent the unbalance of torque in a single coaxial rotors system. In addition, for the same rotation speed, the thrust generated was lesser when both propellers rotates in the same direction compared to when each propeller rotated in the opposite directions of each other. Therefore, the counter-rotating coaxial rotors system is more efficient in generating thrust.

\section{Acknowledgement}

The authors wish to express sincere gratitude to Ministry of Higher Education and Universiti Tun Hussein Onn Malaysia for the financial support through the "Geran Kontrak Kementerian" research grant (ID: K136).

\section{References}

[1] O. Shea, “A Short History of Unmanned Aerial Vehicles.” [Online]. Available: https://consortiq.com/mediacentre/blog/short-history-unmanned-aerial-vehicles-uavs. [Accessed: 30-Nov-2019]

[2] E. Smith, "Types of Drones - Explore the Different Types of UAV's," February 3, 2017. [Online]. Available: http://www.circuitstoday.com/types-of-drones. [Accessed: 27-Nov-2019]

[3] L. King, "Boeing's cargo UAV a shot in the arm for drone delivery market [VIDEO] | Air Cargo World," 11 January, 2018. [Online]. Available: https://aircargoworld.com/allposts/boeings-cargo-uav-a-shot-in-the-armfor-drone-delivery-market-video/. [Accessed: 30-Nov-2019]

[4] "UTHM creates first cargo drone in Malaysia | Daily Metro." [Online]. Available: https://www.hmetro.com.my/mutakhir/2019/09/497481/uthm-cipta-dron-kargo-pertama-di-malaysia. [Accessed: 29-Nov-2019]

[5] T. Frank, "What Is a Tricopter, Quadcopter, Hexacopter \& Octocopter - Droners Guides," March $19,2018$. [Online]. Available: https://www.dronersguides.com/copters/. [Accessed: 27-Nov-2019]

[6] "Hexacopter - Definition \& Benefits - Yuneec." [Online]. Available: https://www.yuneec.com/en_GB/cameradrones/hexacopter.html. [Accessed: 30-Nov-2019]

[7] L. Chen and P. J. Mckerrow, "Modelling the Lama Coaxial Helicopter," 2012

[8] J. G. Leishman and S. Ananthan, “AERODYNAMIC OPTIMIZATION OF A COAXIAL PROPROTOR,” 2006.

[9] S. D. Fernandes, "Performance Analysis of a Coaxial Helicopter in Hover and Performance Analysis of a Coaxial Helicopter in Hover and Forward Flight Forward Flight Scholarly Commons Citation Scholarly Commons Citation," 2017

[10] C. Martins Simões, "Optimizing a Coaxial Propulsion System to a Quadcopter."

[11] Q. Quan, Introduction to multicopter design and control. Springer Singapore, 2017.

[12] "U15XXL KV29_Manned Aircraft_Combo Pack_T-MOTOR Store-Official Store for T-motor drone motor,ESC,Propeller.” [Online]. Available: http://store-en.tmotor.com/goods.php?id=891. [Accessed: 05-Dec2019] 